JOURNAL OF INTEGRAL EQUATIONS

AND APPLICATIONS

Volume 17, Number 2, Summer 2005

\title{
ON THE ANALYTICAL SOLUTIONS OF TWO SINGULAR INTEGRAL EQUATIONS WITH HILBERT KERNELS
}

\author{
G. MONEGATO AND A. STROZZI
}

ABSTRACT. The analytical solution of two singular integral equations with Hilbert kernel of the first and second kind, respectively, is derived from the known solution of the corresponding singular integral equations with Cauchy kernel of the first and second kind by introducing a proper change of variables.

1. Introduction. The representation of all solutions of the singular integral equations

$$
\begin{aligned}
& \int_{-1}^{+1} \frac{\tilde{f}(x)}{y-x} d x=\tilde{g}(y),-1<y<1 \\
& \tilde{f}(y)-\frac{i \lambda}{\pi} \int_{-1}^{+1} \frac{\tilde{f}(x)}{y-x} d x=\tilde{g}(y), \quad-1<y<1
\end{aligned}
$$

where $\lambda$ is real and the integrals are defined in the Cauchy principal value sense, is of key importance in many applications. Among the several authors who have examined this problem, Söhngen, [13, 14], and Tricomi, $[\mathbf{7}, \mathbf{1 6}, \mathbf{1 7}]$, appear to be the first to have obtained fundamental results on this topic. Here we recall some of them.

Theorem 1 (see $[\mathbf{1 3}, \mathbf{1 4}, \mathbf{1 6}]$ ). If in (1.1) $\tilde{g} \in L_{p}, p>1$, then $\tilde{f} \in L_{q}$ for some $q>1$ and necessarily has the form

$$
\tilde{f}(y)=-\frac{1}{\pi^{2}} \frac{1}{\sqrt{1-y^{2}}} \int_{-1}^{+1} \frac{\tilde{g}(x) \sqrt{1-x^{2}}}{y-x} d x+\frac{C}{\sqrt{1-y^{2}}}
$$

where $C$ is an arbitrary constant.

Key words and phrases. Singular integral equations, first and second kind, Hilbert kernel, analytical solution.

This work has been supported by the Ministero dell'Istruzione, dell'Università e della Ricerca of Italy.

Received by the editors on Feb. 1, 2005, and in revised form on April 21, 2005.

Copyright (C)2005 Rocky Mountain Mathematics Consortium 
Theorem 2 (see [14, p. 43]). If in (1.2) $\tilde{g} \in L_{p}, p>1$ and $\lambda$ is a real number with $\lambda \neq \pm 1$, then $\tilde{f} \in L_{q}$ for some $q>1$ and necessarily has the form

$$
\begin{aligned}
& \text { (1.4) } \tilde{f}(y)= \\
& \begin{array}{r}
\frac{1}{1-\lambda^{2}}\left[\tilde{g}(y)+\frac{i \lambda}{\pi \sqrt{1-y^{2}}}\left(\frac{1+y}{1-y}\right)^{i \gamma} \int_{-1}^{+1} \frac{\tilde{g}(x) \sqrt{1-x^{2}}}{y-x}\left(\frac{1-x}{1+x}\right)^{i \gamma} d x\right] \\
\quad+\frac{C}{\sqrt{1-y^{2}}}\left(\frac{1+y}{1-y}\right)^{i \gamma}
\end{array}
\end{aligned}
$$

where $\gamma=(\operatorname{arccoth} \lambda) / \pi$ and $C$ is an arbitrary constant, if $|\lambda|>1$, while

$$
\begin{aligned}
& \quad \tilde{f}(y)= \\
& \frac{1}{1-\lambda^{2}}\left[\tilde{g}(y)+\frac{i \lambda}{\pi(1-y)}\left(\frac{1+y}{1-y}\right)^{i \gamma} \int_{-1}^{+1} \frac{\tilde{g}(x)}{y-x}(1-x)\left(\frac{1-x}{1+x}\right)^{i \gamma} d x\right] \\
& \quad-i \frac{\sinh 2 \pi \gamma}{2 \pi(1-y)}\left(\frac{1+y}{1-y}\right)^{i \gamma} \int_{-1}^{+1}\left(\frac{1-x}{1+x}\right)^{i \gamma} \tilde{g}(x) d x
\end{aligned}
$$

where $\gamma=(\operatorname{arctanh} \lambda) / \pi$, if $|\lambda|<1$. If $\lambda= \pm 1$, equation (1.2) is in general not solvable in $L_{q}, q>1$.

Here and in the following, for notational convenience we use the same symbol $C$ to denote arbitrary complex constants which in general do not coincide.

Comparatively lower attention seems to have been paid to the twin integral equations

$$
\begin{aligned}
\int_{-\alpha}^{+\alpha} \frac{f(\omega)}{\tan (\theta-\omega)} d \omega & =g(\theta), \quad-\alpha<\theta<\alpha \\
f(\theta)-\frac{i \lambda}{\pi} \int_{-\alpha}^{+\alpha} \frac{f(\omega)}{\tan (\theta-\omega)} d \omega=g(\theta), & -\alpha<\theta<\alpha
\end{aligned}
$$

when $0<\alpha<\pi / 2$, which are of interest in contact problems, see for example [8], where the support is given by two symmetric circular arcs, 
each of length $2 \alpha<\pi$. Indeed, to our knowledge, only the solution of (1.6) has been given, although a proof of it could not be traced in the references quoted in this work and in classical textbooks on singular integral equations. We also notice that for the case $\alpha=\pi / 2$ the singular operator defined in (1.6) is discussed in [11].

The purpose of this note is to prove for (1.6) and (1.7) the analogous results of Theorems 1 and 2 above. This study has been motivated by the circumstance that the equations here examined are not covered in standard textbooks on integral equations, e.g., $[\mathbf{1}, \mathbf{1 0}]$, although such equations, or closely related formulations, are encountered in periodic problems of the theory of elasticity, e.g., $[\mathbf{8}, \mathbf{9}]$.

In Section 2 we will characterize the solutions of equation (1.6), while in Section 3 we will obtain the analogous result for equation (1.7).

In the following, kernels of the type $1 /(x-y)$ and $1 / \tan (\theta-\omega)$ will be referred to as Cauchy and Hilbert kernels, respectively, and the corresponding integrals will be defined in the Cauchy principal value sense.

2. The integral equation of the first kind. The singular integral equation of the first kind with Hilbert kernel examined in this paper is

$$
\int_{-\alpha}^{+\alpha} \frac{f(\omega)}{\tan (\theta-\omega)} d \omega=g(\theta)
$$

where $g \in L_{p}, p>1,0<\alpha<\pi / 2$, and $f$ and $g$ are real unknown and known functions, respectively. The solution of equation (2.1) is known in analytical form, e.g., $[\mathbf{2}, \mathbf{3}, \mathbf{1 8}]$

$$
\begin{aligned}
f(\theta)= & \frac{C \cos \theta}{\sqrt{\sin ^{2} \alpha-\sin ^{2} \theta}}-\frac{1}{\pi^{2} \sqrt{\sin ^{2} \alpha-\sin ^{2} \theta}} \\
& \times \int_{-\alpha}^{+\alpha} \sqrt{\sin ^{2} \alpha-\sin ^{2} \omega} \frac{g(\omega)}{\sin (\theta-\omega)} d \omega
\end{aligned}
$$

where $C$ is an arbitrary constant. The solution reported in [5, p. 365], is unfortunately affected by a misprint.

Although solution (2.2) of equation (2.1) is available, a method for its derivation is briefly presented in order to illustrate the solution procedure also employed for solving the singular integral equation of the 
second kind with Hilbert kernel described in the next section. The solution of the singular integral equation with Hilbert kernel is here derived from the known solution of the parallel singular integral equation with Cauchy kernel, by employing a suitable change of variables.

Consider the singular integral equation of the first kind with Cauchy kernel

$$
\int_{-1}^{+1} \frac{\tilde{f}(x)}{y-x} d x=\tilde{g}(y)
$$

where $\tilde{f}$ and $\tilde{g}$ are real unknown and known functions, respectively. Then introduce the following change of variables [3, p. 316], [8],

$$
\begin{gathered}
x=\frac{\tan \omega}{\tan \alpha} ; \quad y=\frac{\tan \theta}{\tan \alpha} ; \quad d x=\frac{d \omega}{\cos ^{2} \omega \tan \alpha} ; \\
\cos ^{2} \omega=\frac{1}{1+x^{2} \tan ^{2} \alpha} .
\end{gathered}
$$

The integral equation (2.3) with Cauchy kernel thus becomes

$$
\int_{-\alpha}^{+\alpha} \frac{f(\omega)}{(\tan \theta-\tan \omega) \cos ^{2} \omega} d \omega=g(\theta)
$$

where

$$
f(\omega)=\tilde{f}\left(\frac{\tan \omega}{\tan \alpha}\right), \quad g(\theta)=\tilde{g}\left(\frac{\tan \theta}{\tan \alpha}\right)
$$

By employing the trigonometric identity (5.1), the integral equation (2.5) is converted into

$$
\int_{-\alpha}^{+\alpha} \frac{f(\omega)}{\tan (\theta-\omega)} d \omega-\int_{-\alpha}^{+\alpha} f(\omega) \tan \omega d \omega=g(\theta)
$$

or, according to the above change of variables (2.4),

$$
\int_{-\alpha}^{+\alpha} \frac{f(\omega)}{\tan (\theta-\omega)} d \omega-\tan ^{2} \alpha \int_{-1}^{+1} \tilde{f}(x) \frac{x}{1+x^{2} \tan ^{2} \alpha} d x=g(\theta)
$$


By comparing equations (2.1), (2.3) and (2.7), it emerges that it is convenient to abandon equation (2.3) and, instead, to refer to the following singular integral equation with Cauchy kernel

$$
\int_{-1}^{+1} \frac{\tilde{f}(x)}{y-x} d x+\tan ^{2} \alpha \int_{-1}^{+1} \tilde{f}(x) \frac{x}{1+x^{2} \tan ^{2} \alpha} d x=\tilde{g}(y)
$$

since its whole lefthand side is transformed by expressions (2.4) exactly into the lefthand side of equation (2.1), possessing a Hilbert kernel. The solution of the singular integral equation (2.1) with Hilbert kernel will thus be derived from the solution of the singular integral equation (2.8) with Cauchy kernel. By putting

$$
A=\int_{-\alpha}^{+\alpha} f(\omega) \tan \omega d \omega=\tan ^{2} \alpha \int_{-1}^{+1} \tilde{f}(x) \frac{x}{1+x^{2} \tan ^{2} \alpha} d x
$$

where $A$ is an unknown constant depending upon the unknown function $f$, the integral equation (2.8) becomes

$$
\int_{-1}^{+1} \frac{\tilde{f}(x)}{y-x} d x=\tilde{g}(y)-A
$$

whose solution is, according to expression (1.3),

$$
\begin{aligned}
\tilde{f}(y)= & -\frac{1}{\pi^{2}} \frac{1}{\sqrt{1-y^{2}}} \int_{-1}^{+1} \frac{\tilde{g}(x) \sqrt{1-x^{2}}}{y-x} d x \\
& +\frac{C}{\sqrt{1-y^{2}}}+\frac{1}{\pi^{2}} \frac{A}{\sqrt{1-y^{2}}} \int_{-1}^{+1} \frac{\sqrt{1-x^{2}}}{y-x} d x
\end{aligned}
$$

With the aid of integral (5.5), solution (2.11) becomes

$$
\tilde{f}(y)=-\frac{1}{\pi^{2}} \frac{1}{\sqrt{1-y^{2}}} \int_{-1}^{+1} \frac{\tilde{g}(x) \sqrt{1-x^{2}}}{y-x} d x+\frac{C}{\sqrt{1-y^{2}}}+\frac{1}{\pi} \frac{A y}{\sqrt{1-y^{2}}} .
$$

To derive the explicit expression of the solution of the integral equation with Hilbert kernel (2.1), it is necessary to evaluate with expression (2.9) the constant $A$ appearing in (2.12). Noting that

$$
\begin{aligned}
& \int_{-1}^{+1} \frac{1}{\sqrt{1-x^{2}}} \frac{x}{1+x^{2} \tan ^{2} \alpha} d x \int_{-1}^{+1} \frac{\tilde{g}(y) \sqrt{1-y^{2}}}{y-x} d y \\
& =\int_{-1}^{+1} \tilde{g}(y) \sqrt{1-y^{2}} d y \int_{-1}^{+1} \frac{1}{\sqrt{1-x^{2}}} \frac{x}{1+x^{2} \tan ^{2} \alpha} \frac{1}{y-x} d x
\end{aligned}
$$


where the change of the order of integration in this particular case is licit for a function $\tilde{g}$ belonging to $L_{p}, p>1$, see [6, Theorem 4.4], and by exploiting integral (5.6), one obtains

$$
\begin{array}{r}
\int_{-1}^{+1} \frac{1}{\sqrt{1-x^{2}}} \frac{x}{1+x^{2} \tan ^{2} \alpha} d x \int_{-1}^{+1} \frac{\tilde{g}(y) \sqrt{1-y^{2}}}{y-x} d y \\
=-\pi \cos \alpha \int_{-1}^{+1} \tilde{g}(x) \frac{\sqrt{1-x^{2}}}{1+x^{2} \tan ^{2} \alpha} d x
\end{array}
$$

By introducing representation (2.12) into (2.9), and taking advantage of integral (5.7) and formula (2.14), the expression of the constant $A$ becomes, after some straightforward manipulations

$$
A=-\frac{\tan ^{2} \alpha}{\pi} \int_{-1}^{+1} \tilde{g}(x) \frac{\sqrt{1-x^{2}}}{1+x^{2} \tan ^{2} \alpha} d x
$$

The explicit form of solution (2.12) of the integral equation with Cauchy kernel (2.8) thus becomes

$$
\begin{aligned}
\tilde{f}(y)= & -\frac{1}{\pi^{2}} \frac{1}{\sqrt{1-y^{2}}} \int_{-1}^{+1} \frac{\tilde{g}(x) \sqrt{1-x^{2}}}{y-x} d x \\
& -\frac{\tan ^{2} \alpha}{\pi^{2}} \frac{y}{\sqrt{1-y^{2}}} \int_{-1}^{+1} \frac{\tilde{g}(x) \sqrt{1-x^{2}}}{1+x^{2} \tan ^{2} \alpha} d x+\frac{C}{\sqrt{1-y^{2}}}
\end{aligned}
$$

It has already been noted that the change of variables (2.4) transforms the integral equation with Cauchy kernel (2.8) into the integral equation with Hilbert kernel (2.1). Consequently, by applying the same change of variables (2.4) to the solution (2.16) of the integral equation with Cauchy kernel (2.8), the solution of the integral equation with Hilbert kernel (2.1) is obtained

$$
\begin{aligned}
f(\theta) & =\frac{C \tan \alpha}{\sqrt{\tan ^{2} \alpha-\tan ^{2} \theta}}-\frac{1}{\pi^{2} \sqrt{\tan ^{2} \alpha-\tan ^{2} \theta}} \\
& \times \int_{-\alpha}^{+\alpha} g(\omega) \sqrt{\tan ^{2} \alpha-\tan ^{2} \omega}\left[\frac{1}{(\tan \theta-\tan \omega) \cos ^{2} \omega}+\tan \theta\right] d \omega .
\end{aligned}
$$

By exploiting the trigonometric identities (5.2) and (5.3), the more compact expression (2.2) is finally achieved. 
The statement below then follows.

Theorem 3. If in (2.1) we have $g \in L_{p}$ and $p>1$, then $f \in L_{q}$ for some $q>1$ and necessarily has the form (2.17), where $C$ is an arbitrary constant.

3. The integral equation of the second kind. The singular integral equation of the second kind with Hilbert kernel examined in this paper is

$$
f(\theta)-\frac{i \lambda}{\pi} \int_{-\alpha}^{+\alpha} \frac{f(\omega)}{\tan (\theta-\omega)} d \omega=g(\theta) .
$$

For simplicity we only consider the case $|\lambda|>1$, which is of interest in contact problems. The case $|\lambda|<1$ can be treated in the same way, but it requires the analytic evaluation of further integrals like those we have listed in the Appendix.

From an attentive review of the pertinent literature it emerges that an analytical solution may be derived of the integral equation (3.1). In fact, in [15] it is noted that in [9] an equation analogous to (3.1) "has a closed form solution." It is further observed that, to obtain the exact solution, "the whole procedure seems quite complicated and even in this case, where a closed-form solution exists, the numerical solution proposed here seems to be of some value." In [12] a numerical solution of an equation similar to (3.1) is compared to the "exact solution," but an explicit expression for this is not provided. Being the present authors unaware of the availability of the expression of the exact solution for the singular integral equation of the second kind with Hilbert kernel (3.1), the aim of this section is to derive such analytical solution.

As for the first kind analogue, we obtain the explicit solution of the singular integral equation of the second kind with Hilbert kernel (3.1) from the known solution (1.4) of the parallel singular integral equation with Cauchy kernel, by introducing into this latter the change of variables (2.4). One obtains

$$
f(\theta)-\frac{i \lambda}{\pi} \int_{-\alpha}^{+\alpha} \frac{f(\omega)}{(\tan \theta-\tan \omega) \cos ^{2} \omega} d \omega=g(\theta)
$$


or, with the aid of identity (5.1)

$$
\begin{aligned}
f(\theta) & -\frac{i \lambda}{\pi} \int_{-\alpha}^{+\alpha} \frac{f(\omega)}{\tan (\theta-\omega)} d \omega \\
& +\frac{i \lambda}{\pi} \tan ^{2} \alpha \int_{-1}^{+1} \tilde{f}(x) \frac{x}{1+x^{2} \tan ^{2} \alpha} d x=g(\theta) .
\end{aligned}
$$

By comparing equations (3.1), (1.2) and (3.3), it appears that it is convenient to dismiss equation (1.2) and, instead, to consider the following singular integral equation with Cauchy kernel

$\tilde{f}(y)-\frac{i \lambda}{\pi} \int_{-1}^{+1} \frac{\tilde{f}(x)}{y-x} d x-\frac{i \lambda}{\pi} \tan ^{2} \alpha \int_{-1}^{+1} \tilde{f}(x) \frac{x}{1+x^{2} \tan ^{2} \alpha} d x=\tilde{g}(y)$

since its whole lefthand side is transformed by the change of variables (2.4) into the lefthand side of equation (3.1) having a Hilbert kernel. By introducing the constant $A$ already defined in (2.9), the integral equation (3.4) becomes

$$
\tilde{f}(y)-\frac{i \lambda}{\pi} \int_{-1}^{+1} \frac{\tilde{f}(x)}{y-x} d x=\tilde{g}(y)+\frac{i \lambda}{\pi} A
$$

whose solution according to (1.4) is

$$
\begin{aligned}
\tilde{f}(y)= & \frac{1}{1-\lambda^{2}}\left[\tilde{g}(y)+\frac{i \lambda}{\pi} A+\frac{i \lambda}{\pi \sqrt{1-y^{2}}}\left(\frac{1+y}{1-y}\right)^{i \gamma}\right. \\
& \times \int_{-1}^{+1} \frac{\tilde{g}(x) \sqrt{1-x^{2}}}{y-x}\left(\frac{1-x}{1+x}\right)^{i \gamma} d x \\
& \left.-\frac{\lambda^{2} A}{\pi^{2} \sqrt{1-y^{2}}}\left(\frac{1+y}{1-y}\right)^{i \gamma} \int_{-1}^{+1} \frac{\sqrt{1-x^{2}}}{y-x}\left(\frac{1-x}{1+x}\right)^{i \gamma} d x\right] \\
& +\frac{C}{\sqrt{1-y^{2}}}\left(\frac{1+y}{1-y}\right)^{i \gamma}
\end{aligned}
$$

where $C$ is an arbitrary constant. 
By employing (5.8), solution (3.6) becomes

$$
\begin{aligned}
\tilde{f}(y)= & \frac{1}{1-\lambda^{2}}\left[\tilde{g}(y)-\frac{\lambda^{2} A}{\pi \cosh \pi \gamma} \frac{y}{\sqrt{1-y^{2}}}\left(\frac{1+y}{1-y}\right)^{i \gamma}\right. \\
& \left.+\frac{i \lambda}{\pi \sqrt{1-y^{2}}}\left(\frac{1+y}{1-y}\right)^{i \gamma} \int_{-1}^{+1} \frac{\tilde{g}(x) \sqrt{1-x^{2}}}{y-x}\left(\frac{1-x}{1+x}\right)^{i \gamma} d x\right] \\
& +\frac{C}{\sqrt{1-y^{2}}}\left(\frac{1+y}{1-y}\right)^{i \gamma},
\end{aligned}
$$

where the new arbitrary constant $C$ is different from the one in (3.6).

To obtain the explicit expression of the solution to the integral equation with Hilbert kernel (3.1), it is necessary to evaluate with expression (2.9) the constant $A$ appearing in (3.7). Noting that

$$
\begin{aligned}
\int_{-1}^{+1} & \frac{1}{\sqrt{1-y^{2}}}\left(\frac{1+y}{1-y}\right)^{i \gamma} \frac{y}{1+y^{2} \tan ^{2} \alpha} d y \int_{-1}^{+1} \frac{\tilde{g}(x) \sqrt{1-x^{2}}}{y-x}\left(\frac{1-x}{1+x}\right)^{i \gamma} d x \\
= & -\int_{-1}^{+1} \tilde{g}(x) \sqrt{1-x^{2}}\left(\frac{1-x}{1+x}\right)^{i \gamma} d x \\
& \times \int_{-1}^{+1} \frac{1}{\sqrt{1-y^{2}}}\left(\frac{1+y}{1-y}\right)^{i \gamma} \frac{y}{1+y^{2} \tan ^{2} \alpha} \frac{1}{x-y} d y
\end{aligned}
$$

where the applicability of the change of the order of integration follows from Theorem 4.4 in [6], upon exploitation of integral (5.9), expression (3.8) becomes

$$
\begin{aligned}
& \int_{-1}^{+1} \frac{1}{\sqrt{1-y^{2}}}\left(\frac{1+y}{1-y}\right)^{i \gamma} \frac{y}{1+y^{2} \tan ^{2} \alpha} d y \int_{-1}^{+1} \frac{\tilde{g}(x) \sqrt{1-x^{2}}}{y-x}\left(\frac{1-x}{1+x}\right)^{i \gamma} d x \\
& =\frac{\pi}{\cosh \pi \gamma}\left[i \sinh \pi \gamma \int_{-1}^{+1} \tilde{g}(x) \frac{x}{1+x^{2} \tan ^{2} \alpha} d x\right. \\
& \quad+\cos \alpha \cosh 2 \alpha \gamma \int_{-1}^{+1} \frac{\tilde{g}(x) \sqrt{1-x^{2}}}{1+x^{2} \tan ^{2} \alpha}\left(\frac{1-x}{1+x}\right)^{i \gamma} d x \\
& \left.\quad-i \sin \alpha \sinh 2 \alpha \gamma \int_{-1}^{+1} \frac{\tilde{g}(x) x \sqrt{1-x^{2}}}{1+x^{2} \tan ^{2} \alpha}\left(\frac{1-x}{1+x}\right)^{i \gamma} d x\right]
\end{aligned}
$$


By introducing formulae (3.7),(3.9) and integrals (5.10) and (5.11) into expression (2.9), the value of the constant $A$ becomes, after tedious but routine calculations,

$$
\begin{aligned}
A= & -\tan ^{2} \alpha \sinh \pi \gamma \\
& \times\left[\tan \alpha \tanh 2 \alpha \gamma \int_{-1}^{+1} \frac{\tilde{g}(x) x \sqrt{1-x^{2}}}{1+x^{2} \tan ^{2} \alpha}\left(\frac{1-x}{1+x}\right)^{i \gamma} d x\right. \\
& \left.+i \int_{-1}^{+1} \frac{\tilde{g}(x) \sqrt{1-x^{2}}}{1+x^{2} \tan ^{2} \alpha}\left(\frac{1-x}{1+x}\right)^{i \gamma} d x\right]+C \frac{i \pi \tan \alpha \tanh 2 \alpha \gamma}{\cosh \pi \gamma}
\end{aligned}
$$

Upon application of the change of variables (2.4) and of the trigonometric identity (5.4), expression (3.10) of the constant $A$ may be expressed as

$$
\begin{aligned}
A= & -\sinh \pi \gamma \\
& \times\left[\tanh 2 \alpha \gamma \int_{-\alpha}^{+\alpha} g(\omega) \tan \omega \sqrt{\tan ^{2} \alpha-\tan ^{2} \omega}\left(\frac{\sin (\alpha-\omega)}{\sin (\alpha+\omega)}\right)^{i \gamma} d \omega\right. \\
& \left.+i \int_{-\alpha}^{+\alpha} g(\omega) \sqrt{\tan ^{2} \alpha-\tan ^{2} \omega}\left(\frac{\sin (\alpha-\omega)}{\sin (\alpha+\omega)}\right)^{i \gamma} d \omega\right] \\
& +C \frac{i \pi \tan \alpha \tanh 2 \alpha \gamma}{\cosh \pi \gamma} .
\end{aligned}
$$

By applying the change of variables (2.4) to formula (3.7), the analytical expression of the solution to the integral equation (3.1) of the second kind with Hilbert kernel for the case $|\lambda|>1$ is finally achieved

$$
\begin{aligned}
f(\theta)= & -\sinh ^{2} \pi \gamma g(\theta)+\frac{A \cosh \pi \gamma \tan \theta}{\pi \sqrt{\tan ^{2} \alpha-\tan ^{2} \theta}}\left(\frac{\sin (\alpha+\theta)}{\sin (\alpha-\theta)}\right)^{i \gamma} \\
& +C \frac{\tan \alpha}{\sqrt{\tan ^{2} \alpha-\tan ^{2} \theta}}\left(\frac{\sin (\alpha+\theta)}{\sin (\alpha-\theta)}\right)^{i \gamma} \\
& -i \frac{\sinh \pi \gamma \cosh \pi \gamma}{\pi \sqrt{\tan ^{2} \alpha-\tan ^{2} \theta}}\left(\frac{\sin (\alpha+\theta)}{\sin (\alpha-\theta)}\right)^{i \gamma} \\
& \times\left[\int_{-\alpha}^{+\alpha} \frac{g(\omega) \sqrt{\tan ^{2} \alpha-\tan ^{2} \omega}}{\tan (\theta-\omega)}\left(\frac{\sin (\alpha-\omega)}{\sin (\alpha+\omega)}\right)^{i \gamma} d \omega\right. \\
& \left.-\int_{-\alpha}^{+\alpha} g(\omega) \tan \omega \sqrt{\tan ^{2} \alpha-\tan ^{2} \omega}\left(\frac{\sin (\alpha-\omega)}{\sin (\alpha+\omega)}\right)^{i \gamma} d \omega\right]
\end{aligned}
$$


where the constant $A$ is given by expression (3.11).

The following statement then follows.

Theorem 4. If in (3.1), with $|\lambda|>1$, we have $g \in L_{p}, p>1$, then $f \in L_{q}$ for some $q>1$ and has necessarily the form (3.12), where the constant $C$ is arbitrary.

4. Some examples. Three examples are presented, where we assume $|\lambda|>1$. The first example addresses the following homogeneous singular integral equation of the second kind with Hilbert kernel

$$
f(\theta)-\frac{i \lambda}{\pi} \int_{-\alpha}^{+\alpha} \frac{f(\omega)}{\tan (\theta-\omega)} d \omega=0
$$

From equation (3.11) the expression of $A$ becomes

$$
A=C \frac{i \pi \tan \alpha \tanh 2 \alpha \gamma}{\cosh \pi \gamma}
$$

From (3.7) the solution is

$$
\tilde{f}(y)=C\left[\frac{1}{\sqrt{1-y^{2}}}\left(\frac{1+y}{1-y}\right)^{i \gamma}+i \tan \alpha \tanh 2 \alpha \gamma \frac{y}{\sqrt{1-y^{2}}}\left(\frac{1+y}{1-y}\right)^{i \gamma}\right]
$$

which, by exploiting the trigonometric identities (5.2) and (5.4), may be rewritten in terms of trigonometric functions as

$$
f(\theta)=C \frac{\sin \alpha \cos \theta}{\sqrt{\sin ^{2} \alpha-\sin ^{2} \theta}}\left(\frac{\sin (\alpha+\theta)}{\sin (\alpha-\theta)}\right)^{i \gamma}[1+i \tan \theta \tanh 2 \alpha \gamma]
$$

By employing integral (5.9) in which $\alpha=0$ is set, and integrals (5.10), (5.11) and (5.14), it may be shown that solution (4.3) fulfills equation (3.4) with $\tilde{g}=0$ and, therefore, it satisfies the equation with Hilbert kernel (4.1).

The second example considers the following singular integral equation of the second kind with Hilbert kernel

$$
f(\theta)-\frac{i \lambda}{\pi} \int_{-\alpha}^{+\alpha} \frac{f(\omega)}{\tan (\theta-\omega)} d \omega=1
$$


From (3.10) and integrals (5.12) and (5.13), the expression of $A$ becomes

$$
\begin{aligned}
A= & i \pi \tanh \pi \gamma\left(1-\frac{1}{\cos \alpha \cosh 2 \alpha \gamma}-2 \gamma \tan \alpha \tanh 2 \alpha \gamma\right) \\
& +C \frac{i \pi \tan \alpha \tanh 2 \alpha \gamma}{\cosh \pi \gamma} .
\end{aligned}
$$

From representation (3.7) and integral (5.8), the analytical expression of the solution may be written as

$$
\begin{aligned}
\tilde{f}(y)= & -2 \gamma \sinh \pi \gamma \frac{1}{\sqrt{1-y^{2}}}\left(\frac{1+y}{1-y}\right)^{i \gamma}+\sinh \pi \gamma\left(\frac{A \operatorname{coth} \pi \gamma}{\pi}-i\right) \\
& \times \frac{y}{\sqrt{1-y^{2}}}\left(\frac{1+y}{1-y}\right)^{i \gamma}+\frac{C}{\sqrt{1-y^{2}}}\left(\frac{1+y}{1-y}\right)^{i \gamma},
\end{aligned}
$$

from which it follows

$$
\begin{aligned}
f(\theta)= & \frac{\tan \alpha}{\sqrt{\tan ^{2} \alpha-\tan ^{2} \theta}}\left(\frac{\sin (\alpha+\theta)}{\sin (\alpha-\theta)}\right)^{i \gamma} \\
& \times\left[-2 \gamma \sinh \pi \gamma+\frac{\tan \theta}{\tan \alpha} \sinh \pi \gamma\left(\frac{A \operatorname{coth} \pi \gamma}{\pi}-i\right)+C\right] .
\end{aligned}
$$

By employing integral (5.9) in which $\alpha=0$ is set, and integrals (5.10), (5.11) and (5.14), it may be verified that expression (4.6), where $A$ is defined by (4.5), fulfills the integral equation (3.4) in which $\tilde{g}=1$ is set and, therefore, $f(\theta)$ satisfies the equation with Hilbert kernel (4.4).

The third example examines the following Fredholm integral equation of the second kind with Hilbert kernel

$$
f(\theta)-\frac{i \lambda}{\pi} \int_{-\alpha}^{+\alpha} \frac{f(\omega)}{\tan (\theta-\omega)} d \omega=i \frac{\tan \theta}{\tan \alpha} .
$$

From (3.10) and integrals (5.12) and (5.15), the expression of $A$ becomes

$$
\begin{aligned}
A= & -i \pi \tan \alpha \tanh \pi \gamma\left[\frac{\tanh 2 \alpha \gamma}{2}\left(1+4 \gamma^{2}+\frac{2}{\tan ^{2} \alpha}\right)-\frac{2 \gamma}{\tan \alpha}\right] \\
& +C \frac{i \pi \tan \alpha \tanh 2 \alpha \gamma}{\cosh \pi \gamma}
\end{aligned}
$$


The analytical expression of the solution may be finally derived from (3.7) and integral (5.16):

$$
\begin{aligned}
\tilde{f}(y)= & \sinh \pi \gamma \frac{1}{\sqrt{1-y^{2}}}\left(\frac{1+y}{1-y}\right)^{i \gamma} \\
& \times\left[-\left(\frac{1}{2}+2 \gamma^{2}\right)+\left(\frac{A \operatorname{coth} \pi \gamma}{\pi}-2 i \gamma\right) y+y^{2}\right] \\
& +\frac{C}{\sqrt{1-y^{2}}}\left(\frac{1+y}{1-y}\right)^{i \gamma}
\end{aligned}
$$

that is,

$$
\begin{aligned}
& f(\theta)=\frac{\tan \alpha}{\sqrt{\tan ^{2} \alpha-\tan ^{2} \theta}}\left(\frac{\sin (\alpha+\theta)}{\sin (\alpha-\theta)}\right)^{i \gamma} \\
& \times\left\{\sinh \pi \gamma\left[-\left(\frac{1}{2}+2 \gamma^{2}\right)+\left(\frac{A \operatorname{coth} \pi \gamma}{\pi}-2 i \gamma\right) \frac{\tan \theta}{\tan \alpha}+\frac{\tan ^{2} \theta}{\tan ^{2} \alpha}\right]+C\right\}
\end{aligned}
$$

By employing integral (5.9) in which $\alpha=0$ is set, and integrals (5.10), (5.11), (5.14), (5.17) and (5.18), it may be verified that expression (4.10), where $A$ is defined by (4.9), fulfills the integral equation (3.4) in which $\tilde{g}(y)=i y$ is set and, therefore, it satisfies the equation with Hilbert kernel (4.8).

Acknowledgments. The contributions of G. Braidi and C.M. Buño in checking numerically the integrals of the Appendix, for several values of the parameters $\alpha, \gamma$ and the variable $y$, is gratefully acknowledged. The authors also thank the referees for their careful reading of the manuscript.

\section{APPENDIX}

5. Some useful trigonometric identities are listed below.

$$
\begin{gathered}
\frac{1}{(\tan \theta-\tan \omega) \cos ^{2} \omega}=\frac{1}{\tan (\theta-\omega)}-\tan \omega \\
\frac{1}{\tan ^{2} \alpha-\tan ^{2} \omega}=\frac{\cos ^{2} \alpha \cos ^{2} \omega}{\sin ^{2} \alpha-\sin ^{2} \omega}
\end{gathered}
$$




$$
\begin{gathered}
\frac{1}{\sin (\theta-\omega)}-\frac{\cos \theta}{\cos \omega}\left(\frac{1}{\tan (\theta-\omega)}+\tan \theta-\tan \omega\right)=\frac{\tan \omega \cos \theta}{\cos \omega} \\
\frac{\tan \alpha+\tan \theta}{\tan \alpha-\tan \theta}=\frac{\sin (\alpha+\theta)}{\sin (\alpha-\theta)}
\end{gathered}
$$

Some useful integrals are reported below. The integrals whose integrands are complex functions have been evaluated with the aid of the Plemelj formulae, e.g., [1] . They have been derived assuming $|\alpha|<\pi / 2$ and $\gamma$ real. Details are omitted for brevity.

$$
\int_{-1}^{+1} \frac{1}{\sqrt{1-x^{2}}} \frac{x^{2}}{1+x^{2} \tan ^{2} \alpha} d x=\frac{\pi}{\tan ^{2} \alpha}(1-\cos \alpha)=\frac{\pi \cos ^{2} \alpha}{1+\cos \alpha}
$$

$$
\begin{aligned}
\int_{-1}^{+1} \frac{\sqrt{1-x^{2}}}{y-x}\left(\frac{1-x}{1+x}\right)^{i \gamma} d x & \\
& =i \pi \sqrt{1-y^{2}}\left(\frac{1-y}{1+y}\right)^{i \gamma} \tanh \pi \gamma-\pi \frac{2 i \gamma-y}{\cosh \pi \gamma}
\end{aligned}
$$

$$
\begin{aligned}
\int_{-1}^{+1} \frac{1}{\sqrt{1-x^{2}}} & \frac{x}{1+x^{2} \tan ^{2} \alpha}\left(\frac{1+x}{1-x}\right)^{i \gamma} \frac{1}{y-x} d x \\
= & -\frac{i \pi}{\sqrt{1-y^{2}}} \frac{y}{1+y^{2} \tan ^{2} \alpha}\left(\frac{1+y}{1-y}\right)^{i \gamma} \tanh \pi \gamma \\
& \quad-\frac{\pi \cos \alpha \cosh 2 \alpha \gamma}{\cosh \pi \gamma\left(1+y^{2} \tan ^{2} \alpha\right)}+\frac{i \pi y \sin \alpha \sinh 2 \alpha \gamma}{\cosh \pi \gamma\left(1+y^{2} \tan ^{2} \alpha\right)}
\end{aligned}
$$




$$
\int_{-1}^{+1} \frac{1}{\sqrt{1-x^{2}}} \frac{x}{1+x^{2} \tan ^{2} \alpha}\left(\frac{1+x}{1-x}\right)^{i \gamma} d x=\frac{i \pi \cos \alpha \sinh 2 \alpha \gamma}{\tan \alpha \cosh \pi \gamma}
$$

$$
\begin{aligned}
\int_{-1}^{+1} \frac{1}{\sqrt{1-x^{2}}} \frac{x^{2}}{1+x^{2} \tan ^{2} \alpha}\left(\frac{1+x}{1-x}\right)^{i \gamma} d x \\
=\frac{\pi(1-\cos \alpha \cosh 2 \alpha \gamma)}{\tan ^{2} \alpha \cosh \pi \gamma}
\end{aligned}
$$

$$
\begin{aligned}
\int_{-1}^{+1} \frac{x \sqrt{1-x^{2}}}{1+x^{2} \tan ^{2} \alpha}\left(\frac{1-x}{1+x}\right)^{i \gamma} d x & \\
= & \frac{i \pi}{\tan ^{2} \alpha \cosh \pi \gamma}\left(2 \gamma-\frac{\sinh 2 \alpha \gamma}{\sin \alpha}\right)
\end{aligned}
$$

$$
\int_{-1}^{+1} \frac{\sqrt{1-x^{2}}}{1+x^{2} \tan ^{2} \alpha}\left(\frac{1-x}{1+x}\right)^{i \gamma} d x=\frac{\pi(\cosh 2 \alpha \gamma-\cos \alpha)}{\cos \alpha \tan ^{2} \alpha \cosh \pi \gamma}
$$

$$
\int_{-1}^{+1} \frac{1}{\sqrt{1-x^{2}}}\left(\frac{1+x}{1-x}\right)^{i \gamma} \frac{1}{y-x} d x=-\frac{i \pi}{\sqrt{1-y^{2}}}\left(\frac{1+y}{1-y}\right)^{i \gamma} \tanh \pi \gamma
$$

$$
\begin{aligned}
\int_{-1}^{+1} & \frac{x^{2} \sqrt{1-x^{2}}}{1+x^{2} \tan ^{2} \alpha}\left(\frac{1-x}{1+x}\right)^{i \gamma} d x \\
& =\frac{\pi}{2 \tan ^{2} \alpha \cosh \pi \gamma}\left(1+4 \gamma^{2}+\frac{2}{\tan ^{2} \alpha}-2 \frac{\cosh 2 \alpha \gamma}{\sin \alpha \tan \alpha}\right)
\end{aligned}
$$

$$
\begin{aligned}
& \text { (5.16) } \int_{-1}^{+1} x \sqrt{1-x^{2}}\left(\frac{1-x}{1+x}\right)^{i \gamma} \frac{1}{y-x} d x \\
& =i \pi y \sqrt{1-y^{2}}\left(\frac{1-y}{1+y}\right)^{i \gamma} \tanh \pi \gamma+\frac{\pi}{\cosh \pi \gamma}\left(y^{2}-2 i \gamma y-\frac{1+4 \gamma^{2}}{2}\right)
\end{aligned}
$$

$$
\begin{aligned}
& \int_{-1}^{+1} \frac{x^{2}}{\sqrt{1-x^{2}}}\left(\frac{1+x}{1-x}\right)^{i \gamma} \frac{1}{y-x} d x \\
& =-\frac{i \pi y^{2}}{\sqrt{1-y^{2}}}\left(\frac{1+y}{1-y}\right)^{i \gamma} \tanh \pi \gamma-\frac{\pi}{\cosh \pi \gamma}(2 i \gamma+y)
\end{aligned}
$$




$$
\begin{aligned}
\int_{-1}^{+1} \frac{1}{\sqrt{1-x^{2}}} \frac{x^{3}}{1+x^{2} \tan ^{2} \alpha}\left(\frac{1+x}{1-x}\right)^{i \gamma} d x \\
=\frac{i \pi}{\tan ^{2} \alpha \cosh \pi \gamma}\left(2 \gamma-\frac{\cos \alpha \sinh 2 \alpha \gamma}{\tan \alpha}\right)
\end{aligned}
$$

\section{REFERENCES}

1. R. Estrada and R.P. Kanwal, Singular integral equations, Birkhäuser, Boston, 2000 .

2. M.G. El-Sheikh, M.E. Khalifa and V. Gavdzinski, The method of integral equation formulation and the unbounded solutions of elastic contact problems, Comput. Math. Appl. 36 (1998), 33-39.

3. E.I. Grigolyuk and V.M. Tolkachev, Contact problems in the theory of plates and shells, Mir, Moscow, 1987.

4. N.I. Ioakimidis, A natural interpolation formula for the numerical solution of singular integral equations with Hilbert kernel, BIT 23 (1983), 92-104.

5. F.F. Ling, W.M. Lai and Don A. Lucca, Fundamentals of surface mechanics, Springer, New York, 2002.

6. S.G. Mikhlin and S. Prössdorf, Singular integral operators, Akademie-Verlag, Berlin, 1986.

7. G. Monegato, Numerical resolution of the generalized airfoil equation with Possio kernel, in Tricomi's Ideas and Contemporary Applied Mathematics, (Internat. Congress in Honour of F.G. Tricomi (Roma, 28-29 November, Turin, 1-2 December 1997), Atti dei Convegni Lincei, vol. 147, Accad. Naz. Lincei, Roma, 1998, pp. 103-121.

8. G. Monegato and A. Strozzi, On the form of the contact reaction in a solid circular plate simply supported along two antipodal edge arcs and deflected by a central transverse concentrated force, J. Elasticity 68 (2002), 13-35.

9. K.S. Parihar and A.C. Garg, An infinite row of collinear cracks at the interface of two bonded dissimilar elastic half planes, Engrg. Fracture Mech. 7 (1975), 751-759.

10. A.D. Polyanin and A.V. Manzhirov, Handbook of integral equations, CRC Press, Boca Raton, 1998.

11. J. Saranen and G. Vainikko, Periodic integral and pseudodifferential equations with numerical approximation, Springer Monographs Math., Springer-Verlag, Berlin, 2002.

12. M.P. Savruk, E. Mandeci and S. Shkarayev, Singular integral equations of the second kind with generalized Cauchy-type kernels and variable coefficients, Internat. J. Numer. Methods Engrg. 45 (1999), 1457-1570.

13. H. Söhngen, Die Lösugen der Integralgleichung $g(x)=(1 / 2 \pi) \int f(\xi) /(x-\xi) d \xi$ und deren Anwendung in der Tragflügeltheorie, Math. Z. 45 (1939), 245-264.

14. — Zur Theorie der Endlichen Hilbert-Transformation, Math. Z. 60 (1954), 31-51. 
15. P.S. Theocaris and N.I. Ioakimidis, On the numerical solution of a Cauchy type singular integral equations and the determination of stress intensity factors in case of complex singularities, Z. Angew. Math. Phys. 28 (1977), 1085-1098.

16. F.G. Tricomi, On the finite Hilbert transformation, Quart. Appl. Math. 2 (1951), 199-211.

17. - Integral equations, Dover, New York, 1957.

18. Li Xing and Li Zhengxing, Effect of a periodic elastic gasket on periodic cracks, Engrg. Fracture Mech. 46 (1993), 127-131.

Dipartimento di Matematica, Politecnico di Torino, Corso Duca degli Abruzzi 24, 10129 Torino, ItAly

E-mail address: giovanni.monegato@polito.it

Modena University, Via Vignolese 905, Modena, Italy

E-mail address: astrozzi@unimo.it 